\title{
Precision calculation of energy levels for four-valent Si I
}

\author{
R. T. Imanbaeva ${ }^{1,2}$, M. G. Kozlov ${ }^{1,2}$, and E. A. Konovalova ${ }^{1}$ \\ 1 Petersburg Nuclear Physics Institute, Gatchina 188300, Russia \\ 2 St. Petersburg Electrotechnical University "LETI", Prof. Popov Str. 5, St. Petersburg, 197376, Russia
}

Received: October 1, 2018/ Revised version: date

\begin{abstract}
We report results of the calculation of the low-lying levels of neutral Si using a combination of the configuration interaction and many-body perturbation theory (CI+MBPT method). We treat Si I as an atom with four valence electrons and use two different starting approximations, namely $V^{N-2}$ and $V^{N-4}$. We conclude that both approximations provide comparable accuracy, on the level of $1 \%$.
\end{abstract}

\section{INTRODUCTION}

The method, which combines configuration interaction (CI) and many-body perturbation theory (MBPT) was suggested in [1] and described in more details in [2. In this approach, which is known as CI+MBPT method, the electrons in an atom are divided into core and valence electrons. Correlations between valence electrons are treated using CI method. The core-core and core-valence correlation are taken into account using second-order MBPT. During the last two decades this method was used for numerous calculations of many-electron atoms and ions and proved to be very effective for systems with two-three valence electrons (see, e.g. 3/4|5|6|), where this method typically provide an accuracy about $1 \%$, or better. Less often this method was used for systems with four, or more valence electrons $[7 / 8|9| 10|11| 12$ and it is still not clear what accuracy one can expect in this case.

There are several reasons why the accuracy and effectiveness of the method may decrease for the four valent systems. First, the size of the CI space for a given length of the basis set grows exponentially with the number of valent electrons. Therefore, it may be impossible to saturate CI space. Second, in order to start with a sufficiently good initial approximation, one needs to include (at least partly) the field of the valence electrons in the initial potential. This leads to the whole new class of the MBPT diagrams 2 and makes results less stable. Finally, for the four-valent atoms there are effective three-electron interactions. This makes calculations much more difficult 1314 .

In this paper we apply CI+MBPT method to Si atom, which we treat as a four-valent system. We compare results for $V^{N-4}$ and $V^{N-2}$ initial approximations, where $N$ is the total number of electrons. The former approximation includes only the field of the core electrons, while

Send offprint requests to: R.T. Imanbaeva

Correspondence to: raykhan.phys@gmail.com the latter one includes also the field of the two valence $3 s$ electrons. We conclude that both approximations lead to comparable accuracy about one percent. For the two lowest singlet states the accuracy is lower, most likely, because of the cusp effect, which is very difficult to reproduce in the CI calculations.

\section{METHOD}

The CI+MBPT method was realized in a number of computer codes (e.g. 4/7/9]). The version we use here is based on the Hartree-Fock-Dirac (HFD) code [16] and the CI code [17. The whole package was recently published in Ref. [18].

In the CI method, the many-electron wave function with a given total angular momentum $J$ is obtained as a linear combination of the many-electron Slater determinants:

$$
\Psi_{J}=\sum_{i} c_{i} \Phi_{i},
$$

where determinants $\Phi_{i}$ are formed from the valence basis orbitals. In our case these orbitals are calculated by solving HFD equations either in the $V^{N-2}$, or $V^{N-4}$ potential. For the case of the partly filled atomic shells we use the average over the non-relativistic configuration, as described in 16. Here we do not need this as both our potentials correcpond to the closed shells $2 p^{6}$, or $3 s^{2}$. The effective Hamiltonian has the form:

$$
H^{\mathrm{eff}}=H_{1}+H_{2}
$$

where $H_{1}$ represents the one-body part of the Hamiltonian, and $\mathrm{H}_{2}$ represents the two-body residual Coulomb interaction.

In the CI+MBPT method we incorporate core excitations by including perturbation theory terms into the effective Hamiltonian. The one-body part $H_{1}$ is modified to include the correlation potential $\Sigma_{1}$, that accounts for the 
Table 1. Low lying energy levels of Si I in the $V^{N-2}$ approximation [7] [12. The energies (in $\left.\mathrm{cm}^{-1}\right)$ are counted from the ground state. Results of the CI and CI+MBPT calculation are given in columns labeled "CI" and "CI+MBPT". Corresponding relative differences of these two calculations with the experiment [15] are given in percent.

\begin{tabular}{rrrrrrr}
\hline \hline & & & & \multicolumn{2}{c}{ Differences $\%)$} \\
Conf. & Term & Exper. & \multicolumn{1}{c}{ CI } & CI+MBPT & \multicolumn{1}{c}{ CI } & CI+MBPT \\
\hline $3 \mathrm{~s}^{2} 3 \mathrm{p}^{2}$ & ${ }^{3} P_{1}$ & 77 & 77 & 76 & 0.24 & 0.84 \\
$3 \mathrm{~s}^{2} 3 \mathrm{p}^{2}$ & ${ }^{3} P_{2}$ & 223 & 225 & 224 & -0.60 & -0.15 \\
$3 \mathrm{~s}^{2} 3 \mathrm{p}^{2}$ & ${ }^{1} D_{2}$ & 6299 & 6909 & 6469 & -9.69 & -2.71 \\
$3 \mathrm{~s}^{2} 3 \mathrm{p}^{2}$ & ${ }^{1} S_{0}$ & 15394 & 16621 & 15931 & -7.97 & -3.49 \\
$3 \mathrm{~s} 3 \mathrm{p}^{3}$ & ${ }^{5} S_{2}^{o}$ & 33326 & 31472 & 32917 & 5.56 & 1.23 \\
$3 \mathrm{~s}^{2} 3 \mathrm{p} 4 \mathrm{~s}$ & ${ }^{3} P_{0}^{o}$ & 39683 & 39122 & 39773 & 1.41 & -0.23 \\
$3 \mathrm{~s}^{2} 3 \mathrm{p} 4 \mathrm{~s}$ & ${ }^{3} P_{1}^{o}$ & 39760 & 39201 & 39851 & 1.41 & -0.23 \\
$3 \mathrm{~s}^{2} 3 \mathrm{p} 4 \mathrm{~s}$ & ${ }^{3} P_{2}^{o}$ & 39955 & 39395 & 40045 & 1.40 & -0.22 \\
$3 \mathrm{~s}^{2} 3 \mathrm{p} 4 \mathrm{~s}$ & ${ }^{1} P_{1}^{o}$ & 40992 & 40562 & 41136 & 1.05 & -0.35 \\
$3 \mathrm{~s} 3 \mathrm{p}^{3}$ & ${ }^{3} D_{1}^{o}$ & 45276 & 44276 & 45090 & 2.21 & 0.41 \\
$3 \mathrm{~s} 3 \mathrm{p}^{3}$ & ${ }^{3} D_{2}^{o}$ & 45294 & 44295 & 45108 & 2.21 & 0.41 \\
$3 \mathrm{~s} 3 \mathrm{p}^{3}$ & ${ }^{3} D_{3}^{o}$ & 45322 & 44326 & 45138 & 2.20 & 0.41 \\
$3 \mathrm{~s}^{2} 3 \mathrm{p} 4 \mathrm{p}$ & ${ }^{1} P_{1}$ & 47284 & 46536 & 47519 & 1.58 & -0.50 \\
$3 \mathrm{~s}^{2} 3 \mathrm{p} 3 \mathrm{~d}$ & ${ }^{1} D_{2}^{o}$ & 47352 & 46478 & 47314 & 1.84 & 0.08 \\
$3 \mathrm{~s}^{2} 3 \mathrm{p} 4 \mathrm{p}$ & ${ }^{3} D_{1}$ & 48021 & 47306 & 47722 & 1.49 & 0.62 \\
$3 \mathrm{~s}^{2} 3 \mathrm{p} 4 \mathrm{p}$ & ${ }^{3} D_{2}$ & 48102 & 47390 & 47805 & 1.48 & 0.62 \\
$3 \mathrm{~s}^{2} 3 \mathrm{p} 4 \mathrm{p}$ & ${ }^{3} D_{3}$ & 48264 & 47552 & 47966 & 1.48 & 0.62 \\
$3 \mathrm{~s}^{2} 3 \mathrm{p} 4 \mathrm{p}$ & ${ }^{3} P_{0}$ & 49028 & 48381 & 48768 & 1.32 & 0.53 \\
$3 \mathrm{~s}^{2} 3 \mathrm{p} 4 \mathrm{p}$ & ${ }^{3} P_{1}$ & 49061 & 48409 & 48797 & 1.33 & 0.54 \\
\hline \hline
\end{tabular}

core-valence correlations (it is also known as self-energy correction):

$$
H_{1} \rightarrow H_{1}+\Sigma_{1}
$$

and the two-body term $\mathrm{H}_{2}$ now includes the effective screening of the two-body interactions by the core:

$$
H_{2} \rightarrow H_{2}+\Sigma_{2}
$$

Both $\Sigma_{1}$ and $\Sigma_{2}$ are calculated in the second-order MBPT 2. In the same second order of MBPT there is also a three-electron correction to the Hamiltonian $\Sigma_{3}$ [2], which describes effective three-electron interactions between valence electrons induced by the core polarization effects. This interaction is typically very small, but becomes important for partly filled $d$, or $f$ shells 1314 .

\section{RESULTS AND DISCUSSIONS}

The basis set was constructed in the frame of the HFD approach. In the $V^{N-2}$ approximation we start with solution of the Dirac-Fock equations for the $\left[1 \mathrm{~s}^{2} 2 \mathrm{~s}^{2} 2 \mathrm{p}^{6} 3 \mathrm{~s}^{2}\right]$ closed shells. As a next step, all orbitals up $3 s$ were frozen and $4-5 s, 3-5 p, 3-5 d, 4-5 f$, and $5 g$ orbitals were constructed in the same $V^{N-2}$ potential. Higher virtual orbitals were obtained with the help of B-splines 20. The lower components of the Dirac bispinors were formed using kinetic balance condition (see, e.g. [18]). The MBPT corrections to the effective Hamiltonian were calculated in the second order MBPT. Note that in this case we had to include the so-called subtraction diagrams [2].

To illustrate the role of the core-valence correlations we calculated the low-lying energy levels using pure valence $\mathrm{CI}$ in the frosen core approximation and in the frame of the CI+MBPT method. The results of the energy level calculations are presented in Table 1. The energies are counted 
Table 2. The energy levels (in $\mathrm{cm}^{-1}$ ) obtained in $V^{N-2}$ and $V^{N-4}$ approximations are compared with the experiment and the results by Savukov [19]. The energies are counted from the ground state.

\begin{tabular}{ccrrrrrrr}
\hline \hline & \multicolumn{4}{c}{ CI+MBPT } & \multicolumn{4}{c}{ Differences (\%) } \\
Conf. & Term & Exper. & $V^{N-2}$ & $V^{N-4}$ & Ref. [19] & $V^{N-2}$ & $V^{N-4}$ & Ref. [19] \\
\hline $3 \mathrm{~s}^{2} 3 \mathrm{p}^{2}$ & ${ }^{3} P_{1}$ & 77 & 76 & 75 & 80 & 1.30 & 2.60 & -3.90 \\
$3 \mathrm{~s}^{2} 3 \mathrm{p}^{2}$ & ${ }^{3} P_{2}$ & 223 & 224 & 221 & 234 & -0.15 & 0.90 & -4.93 \\
$3 \mathrm{~s}^{2} 3 \mathrm{p} 4 \mathrm{~s}$ & ${ }^{3} P_{0}^{o}$ & 39683 & 39773 & 39669 & 39201 & -0.23 & 0.04 & 1.21 \\
$3 \mathrm{~s}^{2} 3 \mathrm{p} 4 \mathrm{~s}$ & ${ }^{3} P_{1}^{o}$ & 39760 & 39851 & 39747 & 39282 & -0.23 & 0.03 & 1.20 \\
$3 \mathrm{~s}^{2} 3 \mathrm{p} 4 \mathrm{~s}$ & ${ }^{3} P_{2}^{o}$ & 39955 & 40045 & 39941 & 39485 & -0.22 & 0.04 & 1.18 \\
$3 \mathrm{~s}^{2} 3 \mathrm{p} 4 \mathrm{~s}$ & ${ }^{1} P_{1}^{o}$ & 40992 & 41136 & 41003 & 40606 & -0.35 & -0.03 & 0.94 \\
$3 \mathrm{~s} 3 \mathrm{p}^{3}$ & ${ }^{3} D_{1}^{o}$ & 45276 & 45090 & 45144 & 44852 & 0.41 & 0.29 & 0.94 \\
$3 \mathrm{~s}^{2} 3 \mathrm{p} 4 \mathrm{p}$ & ${ }^{1} P_{1}$ & 47284 & 47519 & 47266 & 47611 & -0.50 & 0.04 & -0.69 \\
$3 \mathrm{~s}^{2} 3 \mathrm{p} 4 \mathrm{p}$ & ${ }^{3} D_{1}$ & 48020 & 47722 & 48020 & 48398 & 0.62 & 0.00 & -0.79 \\
\hline \hline
\end{tabular}

from the ground state $\left[3 s^{2} 3 p^{2}\right]^{3} P_{0}$. The values are given in $\mathrm{cm}^{-1}$. The differences of our results with the experimental data from NIST 15 are given in the last columns to illustrate the accuracy of each approach. We find that the accuracy of the CI method is mostly on the order of few percent, while the accuracy of the CI+MBPT method is mostly better than $1 \%$. The lowest relative accuracy is for the two singlet states ${ }^{1} D_{2}$ and ${ }^{1} S_{0}$ of the configuration $3 s^{2} 3 p^{2}$. On the one hand, these levels lye rather close to the ground state and the absolute accuracy for these levels is not so much different from the others. On the other hand, for the singlet states the exact wave function has a cusp at $r_{i k}=0$. To reproduce this cusp accurately one needs very large CI space. Therefore, it is not surprising that we have not saturated CI space for these states.

As discussed above, when we apply the CI+MBPT method to the atoms with more than two valence electrons, the effective valence Hamiltonian includes threeelectron term $\Sigma_{3}$. Here we calculated respective corrections and found them to be smaller than the overall theoretical error. We conclude that three-electron interactions are insignificant for Si I. This is not surprising as the core here does not include $d$, or $f$ shells and the valence $3 d$ orbital is weakly bound and does not overlap with the core.

Recently the new accurate method to account for the QED corrections in the polyvalent systems was suggested in Refs. 21 22]. We calculated these corrections together with Breit corrections to the Coulomb interaction and added them to our final results. In general these contributions appear to be too small to influence our final accuracy. However, they slightly improve theoretical fine structure.

We have also considered Si I as the four-valence atom using $V^{N-4}$ approximation. Calculations were quite similar, with the exception of the construction of the basis set. The latter was formed by solving the Dirac-Fock equations for the $\left[1 \mathrm{~s}^{2} 2 \mathrm{~s}^{2} 2 \mathrm{p}^{6}\right]$ closed shells. Then, the orbitals $4-5 s, 3-5 p, 3-5 d, 4-5 f$, and $5 g$ were constructed in the same $V^{N-4}$ potential. For this potential the MBPT part did not include subtraction diagrams as the potential $V^{N-4}$ corresponds to the bare core. For CI calculations we used exactly the same sets of even- and odd-parity configurations, as for the calculations in the $V^{N-2}$ approximation.

In Table 2 we compare results obtained within the CI+MBPT method in the $V^{N-2}$ and $V^{N-4}$ approximations. For the neutral atom, the initial $V^{N-4}$ approximation is clearly less accurate, than the $V^{N-2}$ approximation. For this reason the CI calculation here gives poorer agreement with the experiment. However, Table 2 demonstrate that the accuracy of the final CI+MBPT results are comparable. In fact, for the majority of states the $V^{N-4}$ approximation is slightly better. Our results found here are in good agreement with previous calculation by Savukov [19], but, in general, a little more accurate.

\section{CONCLUSION}

We have studied the accuracy of the CI+MBPT method for the four-valent Si. We performed calculations in the $V^{N-2}$ and $V^{N-4}$ initial approximations and studied the role of the Breit, QED, and three-electron corrections. Our results show that both initial approximations lead to comparable final accuracy, though the $V^{N-4}$ approximation is a little better. Both our calculations have slightly higher accuracy than recent calculation in Ref. 19. The QED and Breit corrections improve theoretical fine structure, but do not improve the gross structure. The effective three-electron interactions for Si are negligibly small. The overall accuracy of the theory is on the level of $1 \%$. Before including higher order terms of the MBPT it is necessary to saturate the CI space, which appears to be rather costly for the four-valent systems.

This work is partly supported by Russian Foundation for Basic Research Grant No. 14-02-00241.

\section{References}

1. V.A. Dzuba, V.V. Flambaum, M.G. Kozlov, JETP Lett. 63, $882(1996)$ 
2. V.A. Dzuba, V.V. Flambaum, M.G. Kozlov, Phys. Rev. A 54, 3948 (1996)

3. S.G. Porsev, M.G. Kozlov, Y.G. Rakhlina, A. Derevianko, Phys. Rev. A 64, 012508 (2001), arXiv: physics/0102070

4. I.M. Savukov, W.R. Johnson, Phys. Rev. A 65, 042503 (2002)

5. S.G. Porsev, M.S. Safronova, M.G. Kozlov, Phys. Rev. Lett. 108, 173001 arXiv: 1201.5615

6. E.A. Konovalova, M.G. Kozlov, Phys. Rev. A 92, 042508 (2015), 1508.01958

7. V.A. Dzuba, Phys. Rev. A 71, 032512 (2005)

8. V.A. Dzuba, Phys. Rev. A 71, 062501 (2005)

9. J.C. Berengut, Phys. Rev. A 84, 052520 (2011), arXiv:1110.2292

10. M.S. Safronova, V.A. Dzuba, V.V. Flambaum, U.I. Safronova, S.G. Porsev, M.G. Kozlov, Phys. Rev. A 90, 052509 (2014), arxiv: 1409.6124

11. I.M. Savukov, Phys. Rev. A 93, 022511 (2016)

12. S.G. Porsev, M.G. Kozlov, M.S. Safronova, I.I. Tupitsyn, Phys. Rev. A 93, 012501 (2016), 1510.06679

13. J.C. Berengut, V.V. Flambaum, M.G. Kozlov, J. Phys. B 41, 235702 (2008), arXiv: 0806.3501

14. M. Kozlov, M. Safronova, S. Porsev, I. Tupitsyn, Phys. Rev. A 94, 032512 (2016), 1607.05843

15. NIST, Atomic Spectra Database, http://physics.nist.gov/PhysRefData/ASD/index.html

16. V.F. Bratsev, G.B. Deyneka, I.I. Tupitsyn, Bull. Acad. Sci. USSR, Phys. Ser. 41, 173 (1977)

17. S.A. Kotochigova, I.I. Tupitsyn, J. Phys. B 20, 4759 (1987)

18. M. Kozlov, S. Porsev, M. Safronova, I. Tupitsyn, Computer Physics Communications 195, 199 (2015)

19. I.M. Savukov, Phys. Rev. A 91, 022514 (2015)

20. J. Sapirstein, W.R. Johnson, J. Phys. B 29, 5213 (1996)

21. V. Shabaev, I. Tupitsyn, V. Yerokhin, Computer Physics Communications 189, 175 (2015)

22. I.I. Tupitsyn, M.G. Kozlov, M.S. Safronova, V.M. Shabaev, V.A. Dzuba, arXiv (2016), 1607.07064 